\title{
Age-Related Alterations in Signaling Pathways in Articular Chondrocytes: Implications for the Pathogenesis and Progression of Osteoarthritis - A Mini-Review
}

\author{
Peter van der Kraan ${ }^{a} \quad$ Csaba Matta ${ }^{b, d} \quad$ Ali Mobasheri ${ }^{b, c}$ \\ ${ }^{a}$ Department of Rheumatology, Experimental Rheumatology, Radboud University Medical Center, Nijmegen, \\ The Netherlands; ${ }^{b}$ Faculty of Health and Medical Sciences, University of Surrey, Guildford, and 'Arthritis Research \\ UK Centre for Sport, Exercise and Osteoarthritis, Arthritis Research UK Centre for Musculoskeletal Ageing Research, \\ Queen's Medical Centre, Nottingham, UK; ${ }^{\mathrm{d}}$ Department of Anatomy, Histology and Embryology, Faculty of \\ Medicine, University of Debrecen, Debrecen, Hungary
}

\section{Key Words}

Osteoarthritis · Cartilage $\cdot$ Chondrocyte $\cdot$ Aging $\cdot$ Cell signaling $\cdot$ Transforming growth factor- $\beta$. Protein kinase . Phosphoprotein phosphatase

\begin{abstract}
Musculoskeletal conditions are a major burden on individuals, healthcare systems, and social care systems throughout the world, with indirect costs having a predominant economic impact. Aging is a major contributing factor to the development and progression of arthritic and musculoskeletal diseases. Indeed, aging and inflammation (often referred to as 'inflammaging') are critical risk factors for the development of osteoarthritis (OA), which is one of the most common forms of joint disease. The term 'chondrosenescence' has recently been introduced to define the age-dependent deterioration of chondrocyte function and how it undermines cartilage function in OA. An important component of chondrosenescence is the age-related deregulation of subcellular signaling pathways in chondrocytes. This mini-review discusses the role of age-related alterations in
\end{abstract}

\begin{tabular}{ll}
\hline KARGER & $\begin{array}{l}\text { ( 2016 The Author(s) } \\
\text { Published by S. Karger AG, Basel }\end{array}$ \\
$\begin{array}{l}\text { E-Mail karger@karger.com } \\
\text { www.karger.com/ger }\end{array}$ & $\begin{array}{l}\text { This article is licensed under the Creative Commons Attribution- } \\
\text { NonCommercial-NoDerivatives 4.0 International License (CC BY- } \\
\text { NC-ND) (http://www.karger.com/Services/OpenAccessLicense) } \\
\text { Usage and distribution for commercial purposes as well as any dis- } \\
\text { tribution of modified material requires written permission. }\end{array}$
\end{tabular}

chondrocyte signaling pathways. We focus our attention on two major areas: age-dependent alterations in transforming growth factor- $\beta$ signaling and changes in protein kinase and phosphoprotein phosphatase activities in aging chondrocytes. A better understanding of the basic signaling mechanisms underlying aging in chondrocytes is likely to facilitate the development of new therapeutic and preventive strategies for $\mathrm{OA}$ and a range of other age-related osteoarticular disorders.

(c) 2016 The Author(s)

Published by S. Karger AG, Basel

\section{Introduction}

Aging is a risk factor for a variety of chronic health problems, including cancer, diabetes, cardiovascular and neurodegenerative disorders. Advancing age is also a major risk factor for the development of arthritic and musculoskeletal diseases. Osteoarthritis (OA) is an age-relat- 


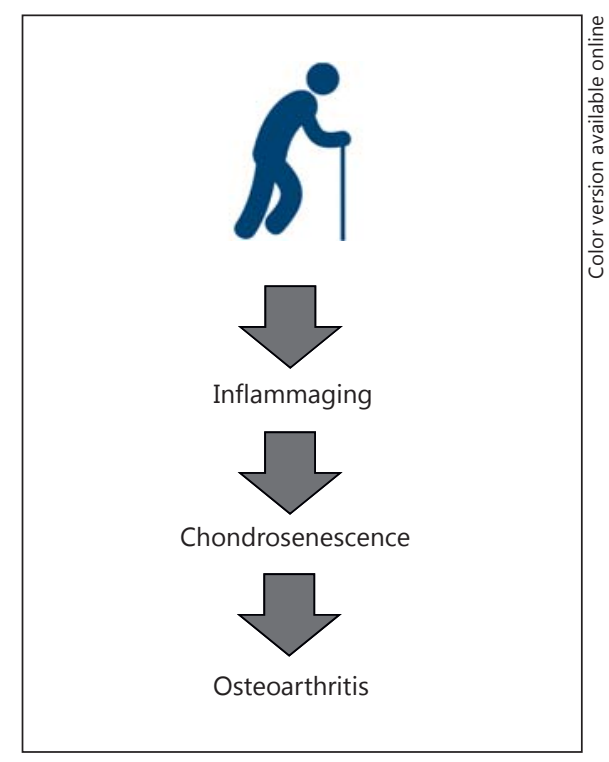

Fig. 1. Aging, inflammaging, and chondrosenescence in OA.

ed disease of synovial joints. It is characterized by the progressive deterioration and loss of articular cartilage with concomitant structural and functional changes in the entire synovial joint, including the synovium, periarticular ligaments, and subchondral bone. OA is actually one of the most common, costly, and disabling forms of joint disease, being far more common than rheumatoid arthritis, psoriatic arthritis, and other forms of joint disease. Age, obesity, and metabolic disease are major risk factors for the development of OA. Traditionally, OA was considered a noninflammatory 'wear and tear' disease. However, over the last few decades, this outdated view has been challenged with solid scientific and clinical evidence. OA is now generally accepted to be an inflammatory and biomechanical whole-organ disease that is influenced by a number of factors including 'inflammaging'. One of the hallmarks of inflammaging is cellular senescence. Evidence from the published literature supports the concept that 'chondrocyte senescence' or 'chondrosenescence' affects the functional phenotype of the cells, is intimately linked with 'inflammaging', and is involved in the pathogenesis and progression of OA [1] (fig. 1). This paper focuses on aging of chondrocytes in OA and the role of age-related alterations in chondrocyte signaling pathways. We turn our attention to the molecular mechanisms and subcellular pathways implicated in chondrocyte aging. We discuss two major areas of research: agedependent alterations in transforming growth factor- $\beta$ (TGF- $\beta$ ) signaling and changes in protein kinase and phosphoprotein phosphatase activities in aging chondrocytes and review the evidence supporting changes in these processes.

\section{Age-Dependent Alterations in TGF- $\beta$ Signaling in OA}

Active TGF- $\beta$ signaling is vital for the physiological maintenance of articular cartilage. Binding of TGF- $\beta$ to its constitutively active type II receptor recruits the type I receptor, and the latter is thereafter phosphorylated and activated, a process that is necessary for TGF- $\beta$-mediated responses. The crucial role of active TGF- $\beta$ signaling is highlighted by the promotion of chondrocyte hypertrophic differentiation and early OA development in mice expressing a truncated, kinase-defective TGF- $\beta$ type II receptor in skeletal tissue [2]. Moreover, the TGF- $\beta$ signaling protein Smad3 is absolutely essential for blocking hypertrophy in articular chondrocytes, both in mice and men $[3,4]$. The presence of normal TGF- $\beta$ signaling is crucial for the maintenance of articular cartilage; however, exposure of joint tissues in animal models to high active TGF- $\beta$ levels leads to osteophyte formation, synovial fibrosis and most likely contributes to joint pain [5-8].

Crystals are common in OA joints and are thought to contribute to cartilage degradation and joint inflammation [9]. Moreover, increased mineralization of the cartilage matrix can occur in OA [10]. TGF- $\beta$ is a known stimulant of inorganic pyrophosphate, a molecule essential for crystal formation. Work on porcine explants has shown that TGF- $\beta$ strongly stimulates inorganic pyrophosphate elaboration in aged cartilage compared to young tissue [11]. A similar finding was reported comparing different human age groups (15-55 and 56-91 years) [12]. These results indicate that age-dependent changes in TGF- $\beta$ responsiveness might contribute to crystal formation in aging joints.

Binding of TGF- $\beta$ to its type II receptor results in the recruitment and phosphorylation of a type I receptor. In chondrocytes, TGF- $\beta$ not only signals via the canonical type Ireceptor ALK5 (TGFBR1) but also via the ALK1 (ACVRL1) receptor $[13,14]$. Signaling via ALK5 leads to activation of the Smad2/3 route, while ALK1 phosphorylation activates the Smad1/5/8 pathway. Using in vitro models, it has been shown that augmented expression of ALK1 increases matrix metalloproteinase-13 (MMP13) expression, while its inhibition reduces MMP13 expression. Moreover, reduced ALK5 expression elevates MMP13 expression levels. In human OA cartilage, ALK1 expression levels correlate positively with MMP13 expression [13]. These data indicate 
that the balance between ALK5 and ALK1 is crucial to maintain cartilage homeostasis and that loss of ALK5 signaling will result in loss of cartilage integrity.

In classic murine studies carried out in the 1990s, the important observation was made that TGF- $\beta$ effects on old cartilage are different from those on young cartilage [15]. TGF- $\beta$ is able to counteract the effects of proinflammatory cytokine interleukin- $1 \beta$ (IL- $1 \beta$ ) only in cartilage of young mice [16]. Aging is associated with a decreased expression of both ALK5 and phosphorylated Smad $2 / 3$ in articular cartilage which was in line with the observation that TGF- $\beta$ does not block IL-1 effects in aged cartilage [17]. Since TGF- $\beta$ can signal via either ALK5 or ALK1, investigation of the age-related changes in expression of ALK5 and ALK1 revealed that the ALK1/ALK5 ratio is significantly increased in old $\mathrm{C} 57 \mathrm{Bl} / 6$ mice compared to young, favoring TGF- $\beta$ signaling via the Smad $1 / 5 / 8$ route and the differentiation of chondrocytes into cells with an autolytic phenotype [13]. This observed change in the ALK1/ALK5 ratio has also been confirmed by others, for instance in the C57/BL1/6 (ICRFa) mouse strain and Dunkin-Hartley guinea pigs [18, 19]. The latter study [19] also demonstrated a progressive switch of the expression of phosphorylated Smad2/3 to Smad1/5/8 in old animals.

Loading of articular cartilage is absolutely essential for its physiological maintenance. Reduced joint loading leads to cartilage degeneration, both in humans and animal models, and patients with spinal cord injuries exhibit progressive loss of knee cartilage. Loading of chondrocytes has been shown to activate $\mathrm{Smad} 2 / 3$ signaling in these cells [20]. Compressive loading of bovine cartilage explants upregulates the expression of $S \operatorname{mad} 2 / 3$ response genes bSerpine1, bSmad7, and bAlk5 while the expression of the Smad1/5/8 responsive gene Id 1 is downregulated [21]. However, when comparing the responsiveness of young (6-36 months) and old (6-13 years) bovine cartilage, it was found that aged cartilage showed a highly reduced capacity for mechanically mediated activation of Smad2/3P signaling when compared to young cartilage [22]. This reduced responsiveness can be a result of a stiffer matrix of old cartilage which makes it harder to mechanically activate TGF- $\beta$ signaling, or is the consequence of an age-related reduction in expression of ALK5, as is observed in aged bovine cartilage. It can also be that reduced ALK5 expression itself is the result of a stiffer matrix, since ALK5 expression is regulated by active TGF- $\beta$ signaling. Irrespective of the underlying cause, it can be expected that an age-related decrease in TGF- $\beta$ signaling (Smad2/3) might impair cartilage preservation, since intact $S m a d 2 / 3$ signaling is crucial for healthy cartilage. The

Age-Related Alterations in Signaling Pathways in Articular Chondrocytes age-related loss of Smad2/3 signaling can be considered a normal part of the aging process, but this can make articular cartilage more vulnerable to OA development at an advanced age compared to young individuals.

$A$ very interesting observation with regard to TGF- $\beta$ signaling and $\mathrm{OA}$ is the relationship between aging and circadian rhythms. Aging is associated with a loss of the normal circadian rhythm [23]. Recent studies have demonstrated that expression of the core clock transcription factor BMAL1 is disrupted in human OA cartilage and in aged mouse cartilage [24]. Deficiency of BMAL1 in mice reduced the levels of phosphorylated $\operatorname{Smad} 2 / 3$ but increased phosphorylated Smad1/5/8 levels. These results link aging and loss of the circadian rhythm to a shift in the ALK5/ALK1 ratio, which may be related to a loss of articular cartilage homeostasis and OA. All above-described observations point to a role of age-related changes in TGF- $\beta$ signaling as a driving force of chondrocyte hypertrophy and OA development and progression. Figure 2 summarizes the alterations in TGF- $\beta$, ALK1/5, and Smad1/5/8 pathways in chondrosenescence and OA.

\section{Alterations in Protein Kinase and Phosphoprotein Phosphatase Activities in Aging Chondrocytes}

Reversible protein phosphorylation on specific Ser/ Thr residues regulated by the balanced activities of protein kinases and phosphoprotein phosphatases is a key process that controls all stages and aspects of the chondrocyte life cycle in response to extracellular stimuli [25, 26]. Amongst the key chondrocyte signaling pathways are protein kinase $\mathrm{A}$, protein kinase $\mathrm{C}$, mitogen-activated protein kinases (MAPKs, such as p38, JNK, and ERK), the phosphoinositide-3 kinase (PI3K)/Akt pathway, as well as protein phosphatase 1A (PP1A), PP2A, and calcineurin (PP2B) [27]. Accumulating evidence suggests that some of these signaling pathways are differentially regulated during the aging process in chondrocytes. It is generally accepted that reactive oxygen species (ROS) contribute to senescence not only by causing direct damage to proteins, lipids, and DNA, but also by modulating cell signaling pathways that promote senescence through activation of redox-sensitive kinases and inhibition of redox-sensitive phosphatases [28]. Increased ROS levels can also upregulate proinflammatory cytokines and MMPs, factors that coconspire and mediate cartilage matrix degradation [29]. Given their central role in chondrosenescence [1], age-related alterations in the MAPK pathways are likely to be particularly important. Several stud- 


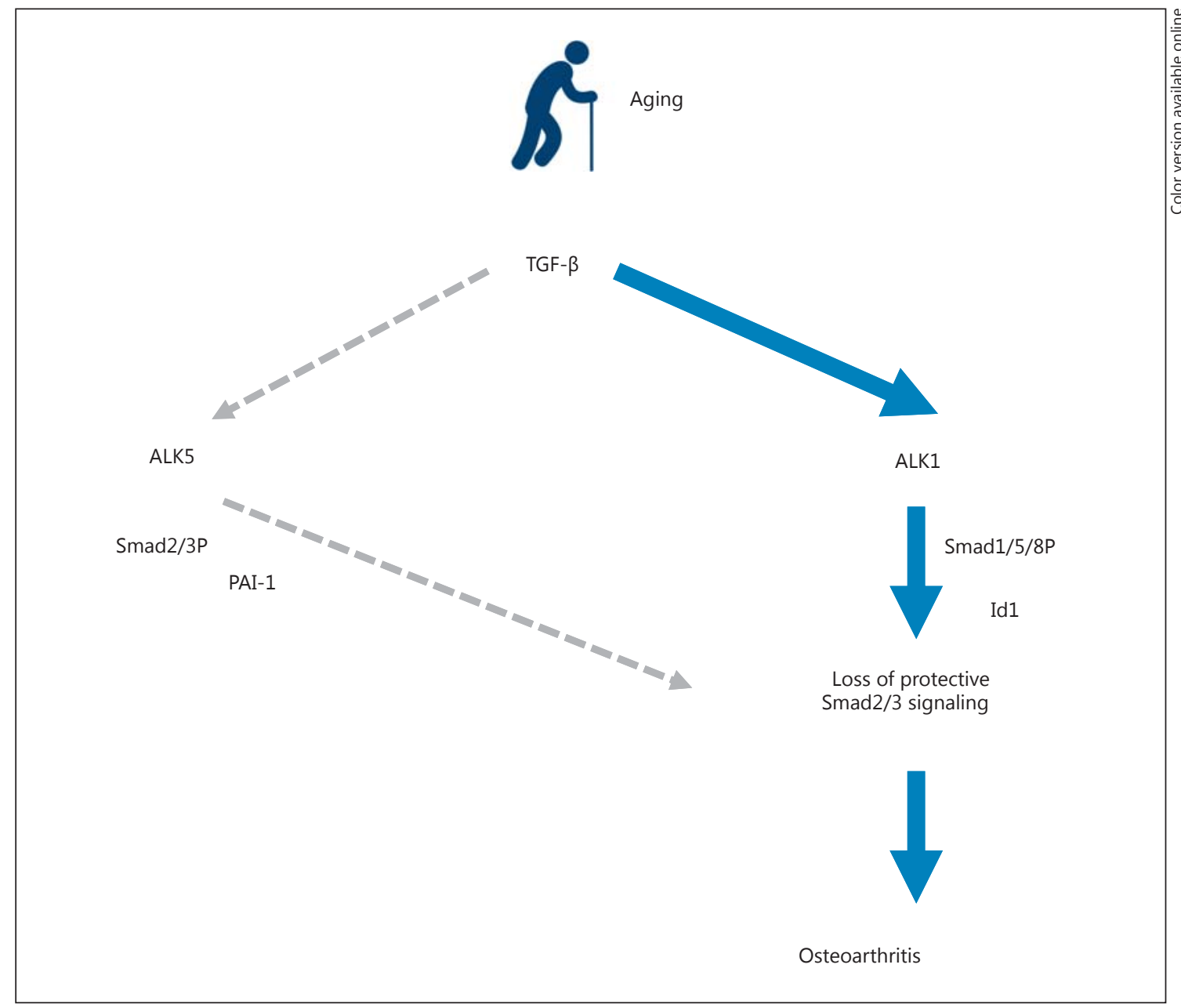

Fig. 2. Alterations in TGF- $\beta, A L K 1 / 5$, and $\operatorname{Smad} 1 / 5 / 8$ pathways in chondrocyte aging. Chondrosenescence results in a switch from the ALK5 to ALK1 pathway leading to the loss of protective Smad2/3 signaling in OA.

ies have suggested the involvement of MAPKs in mediating chondrosenescence. Caveolin-1, the integral membrane protein that serves as a scaffold and can regulate cell signaling pathways, is involved in chondrosenescence; it has been reported to play a role in premature chondrocyte senescence induced by IL- $1 \beta$ and oxidative stress through the activation of 38 MAPK in human and rat models [30]. Elevated levels of ROS can also contribute to reduced proteoglycan synthesis by causing an imbalance in the activity of the PI3K/Akt and the ERK MAPK pathway in human chondrocytes, which have opposing roles in cartilage matrix production [31]. Since the PI3K/Akt pathway plays important roles in regulating the chondrocyte cell cycle, proliferation, differentiation and apoptosis [32], and its activation increases proteoglycan synthesis in human chondrocytes [33], the age-related modulation of its activity may be an important contributor to chondrosenescence. Excessive ROS may also inhibit the insulin signaling pathway through activation of the stress-induced JNK in a human in vitro model [34].

Senescent cells are known to exhibit altered activity and expression of regulatory proteins that control the cell cycle, including p53 and the cyclin-dependent kinase inhibitors $\mathrm{p} 21^{\mathrm{CIP1}}$ and $\mathrm{p} 16^{\mathrm{INK} 4 \mathrm{~A}}$ [35]. Oxidative stress-induced $\mathrm{PKC} \delta$ activation was reported to cooperate with the p $16^{\text {INK4A }}$ retinoblastoma protein pathway in human cells to promote senescence [36]. Oxidative stress is also known to reduce the activity of the calcium-calmodulin-dependent phosphoprotein phosphatase calcineurin, which interferes with the ERK pathway and Sox9 phosphorylation in a chicken micromass model [37]. AMP-activated protein kinase (AMPK), a highly conserved sensor of in- 


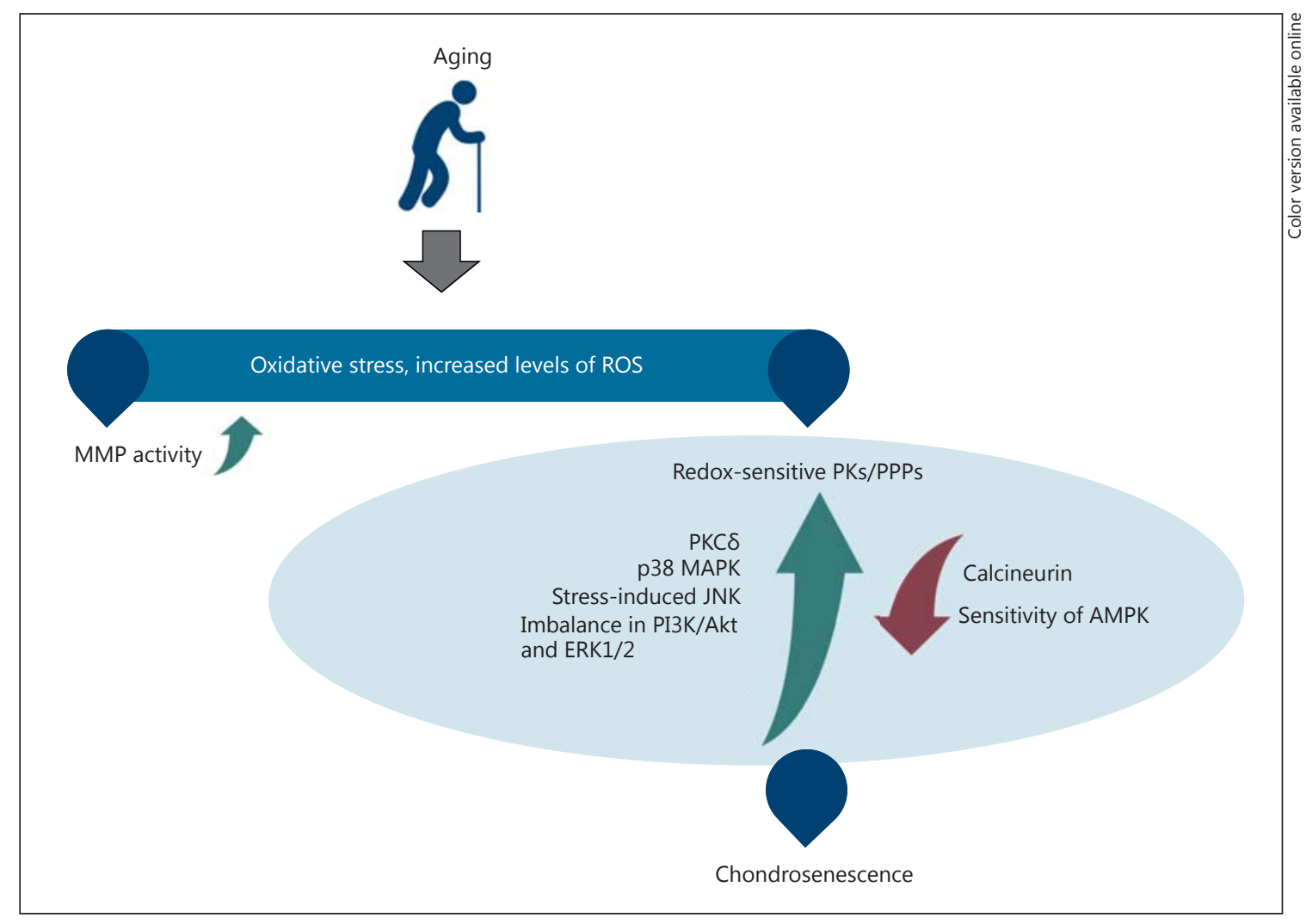

Fig. 3. Effects of chondrocyte aging on redox-sensitive protein kinase and phosphoprotein phosphatase signaling pathways. Oxidative stress and increased levels of ROS stimulate inflammatory and catabolic pathways, upregulate MMPs, and alter the activity of signaling pathways in chondrocytes. PPPs = Phosphoprotein phosphatases.

creased levels of AMP and ADP originating from ATP depletion [38], also has a role in aging as its sensitivity is reduced in aging tissues [39]. Since a number of upstream protein kinases and phosphoprotein phosphatases are involved in its regulation, it is possible that there are agerelated changes in the function of protein phosphatases, i.e. PP2A or PP2Ca, which could suppress AMPK activation with aging [39]. Given its key role as an energy sensor in chondrocytes [40], AMPK is an emerging new target for preventing chondrosenescence and OA. Figure 3 summarizes our current understanding of the effects of chondrocyte aging on redox-sensitive protein kinase and phosphoprotein phosphatase signaling pathways.

\section{Conclusions and Future Directions}

The incidence of OA is steadily rising across the world as the aging population grows. By 2037, it is estimated that the proportion of the population aged $70-90$ years and beyond will expand significantly. Indeed, by the middle of this century, the proportion of centenarians in the United Kingdom and Europe will grow by more than 10fold. Therefore, health and social care systems across the globe need to prepare for the oncoming 'tsunami' of OA cases in the coming decades. Although aging is a nonmodifiable risk factor for the development of OA, there is clinical evidence to suggest that the risk for developing OA can be mitigated and reduced by lifestyle changes that involve weight management (i.e. weight loss and calorie restriction, avoiding obesity/overweight) and maintaining high levels of mobility, thus avoiding the sedentary lifestyles that have become the root cause of most of the world's healthcare issues. At the present time, there are no pharmacological agents available for the treatment of OA. A better understanding of the basic cell signaling mechanisms underlying aging in articular chondrocytes and other cell types in the joint is likely to facilitate the development of new therapeutic strategies for OA, specifically the development of disease-modifying osteoar- 
thritis drugs. In this regard, research on TGF- $\beta$ signaling is more advanced than work on protein kinase and phosphoprotein phosphatase pathways in chondrocytes, and much more research is needed using both human and animal models to understand how calcium signaling and kinase/phosphatase activities are implicated in chondrocyte senescence in the context of OA pathogenesis and progression. Understanding deregulated cell signaling mechanisms in chondrocyte aging may also reveal therapeutic targets and pathways for a range of other age-related osteoarticular disorders. Aging is inevitable, but age-related diseases may be modifiable as long as we have a firm understanding of the molecular gerontology of chondrosenescence in OA. Future research should focus on humans and suitable animal models in order to develop a unified 'one health' therapeutic solution to OA.

\section{Acknowledgements}

The authors wish to acknowledge current and previous members of their laboratories and their internal and external collaborators for contributions. We wish to apologize to those authors whose work could not be included in this focused mini-review.

\section{Disclosure Statement}

A.M. is coordinator of the D-BOARD Consortium funded by European Commission Framework 7 programme (EU FP7; HEALTH.2012.2.4.5-2, project number 305815; Novel Diagnostics and Biomarkers for Early Identification of Chronic Inflammatory Joint Diseases) and member of the Arthritis Research UK Centre for Sport, Exercise, and Osteoarthritis, funded by Arthritis Research UK (grant reference: 20194). A.M. is a member of the Applied Public-Private Research enabling OsteoArthritis Clinical Headway (APPROACH) consortium, a 5-year project funded by the European Commission's Innovative Medicines Initiative. APPROACH is a public-private partnership directed towards osteoarthritis biomarker development through the establishment of a heavily phenotyped and comprehensively analyzed longitudinal cohort. The research leading to these results has received partial support from the Innovative Medicines Initiative Joint Undertaking under grant agreement No. 115770, resources of which are composed of financial contribution from the European Union's Seventh Framework programme (FP7/2007-2013) and EFPIA companies' in kind contribution. P.v.d.K. is a scientific advisor to the APPROACH consortium. The funders had no role in study design, data collection and analysis, decision to publish, or preparation of the manuscript. C.M. is supported by the European Union through a Marie Curie Intra-European Fellowship for career development (project number 625746; acronym: CHONDRION; FP7-PEOPLE2013-IEF).

\section{References}

1 Mobasheri A, Matta C, Zákány R, Musumeci G: Chondrosenescence: definition, hallmarks and potential role in the pathogenesis of osteoarthritis. Maturitas 2015;80:237-244.

-2 Serra R, Johnson M, Filvaroff EH, LaBorde J, Sheehan DM, Derynck R, et al: Expression of a truncated, kinase-defective TGF-beta type II receptor in mouse skeletal tissue promotes terminal chondrocyte differentiation and osteoarthritis. J Cell Biol 1997;139:541-552.

-3 Yang X, Chen L, Xu X, Li C, Huang C, Deng CX: TGF-beta/Smad3 signals repress chondrocyte hypertrophic differentiation and are required for maintaining articular cartilage. J Cell Biol 2001;153:35-46.

-4 Li TF, Gao L, Sheu TJ, Sampson ER, Flick LM, Konttinen YT, et al: Aberrant hypertrophy in Smad3-deficient murine chondrocytes is rescued by restoring transforming growth factor beta-activated kinase 1/activating transcription factor 2 signaling: a potential clinical implication for osteoarthritis. Arthritis Rheum 2010;62:2359-2369.

5 Remst DF, Blom AB, Vitters EL, Bank RA, van den Berg WB, Blaney Davidson EN, et al: Gene expression analysis of murine and human osteoarthritis synovium reveals elevation of transforming growth factor $\beta$-responsive genes in osteoarthritis-related fibrosis. Arthritis Rheum 2014;66:647-656.
6 Van Beuningen HM, van der Kraan PM, Arntz OJ, van den Berg WB: Transforming growth factor-beta 1 stimulates articular chondrocyte proteoglycan synthesis and induces osteophyte formation in the murine knee joint. Lab Invest 1994;71:279-290.

-7 Scharstuhl A, Vitters EL, van der Kraan PM, van den Berg WB: Reduction of osteophyte formation and synovial thickening by adenoviral overexpression of transforming growth factor beta/bone morphogenetic protein inhibitors during experimental osteoarthritis. Arthritis Rheum 2003;48:3442-3451.

8 Blaney Davidson EN, van Caam AP, Vitters EL, Bennink MB, Thijssen E, van den Berg $\mathrm{WB}$, et al: TGF- $\beta$ is a potent inducer of nerve growth factor in articular cartilage via the ALK5-Smad2/3 pathway. Potential role in $\mathrm{OA}$ related pain? Osteoarthritis Cartilage 2015;23:478-486.

-9 Rosenthal AK: Articular cartilage vesicles and calcium crystal deposition diseases. Curr Opin Rheumatol 2016;28:127-132.

-10 Fuerst M, Bertrand J, Lammers L, Dreier R, Echtermeyer F, Nitschke Y, et al: Calcification of articular cartilage in human osteoarthritis. Arthritis Rheum 2009;60:2694-2703.
Rosenthal AK, Ryan LM: Ageing increases growth factor-induced inorganic pyrophosphate elaboration by articular cartilage. Mech Ageing Dev 1994;75:35-44.

12 Rosen F, McCabe G, Quach J, Solan J, Terkeltaub R, Seegmiller JE, et al: Differential effects of aging on human chondrocyte responses to transforming growth factor beta: increased pyrophosphate production and decreased cell proliferation. Arthritis Rheum 1997;40:12751281.

13 Blaney Davidson EN, Remst DF, Vitters EL, van Beuningen $\mathrm{HM}$, Blom $\mathrm{AB}$, Goumans $\mathrm{MJ}$, et al: Increase in ALK1/ALK5 ratio as a cause for elevated MMP-13 expression in osteoarthritis in humans and mice. J Immunol 2009; 182:7937-7945.

14 Finnson KW, Parker WL, ten Dijke P, Thorikay M, Philip A: ALK1 opposes ALK5/Smad3 signaling and expression of extracellular matrix components in human chondrocytes. J Bone Miner Res 2008;23:896-906.

15 Van Beuningen HM, van der Kraan PM, Arntz OJ, van den Berg WB: In vivo protection against interleukin-1-induced articular cartilage damage by transforming growth factor-beta 1: age-related differences. Ann Rheum Dis 1994;53:593-600. 
16 Scharstuhl A, van Beuningen HM, Vitters EL, van der Kraan PM, van den Berg WB: Loss of transforming growth factor counteraction on interleukin 1 mediated effects in cartilage of old mice. Ann Rheum Dis 2002;61:10951098.

17 Blaney Davidson EN, Scharstuhl A, Vitters EL, van der Kraan PM, van den Berg WB: Reduced transforming growth factor-beta signaling in cartilage of old mice: role in impaired repair capacity. Arthritis Res Ther 2005;7:R1338-R1347.

18 Zhao W, Wang T, Luo Q, Chen Y, Leung VY, Wen C, et al: Cartilage degeneration and excessive subchondral bone formation in spontaneous osteoarthritis involves altered TGF- $\beta$ signaling. J Orthop Res 2016;34:763-770.

$\checkmark 19$ Hui W, Young DA, Rowan AD, Xu X, Cawston TE, Proctor CJ: Oxidative changes and signalling pathways are pivotal in initiating age-related changes in articular cartilage. Ann Rheum Dis 2016;75:449-458.

20 Bougault C, Aubert-Foucher E, Paumier A, Perrier-Groult E, Huot L, Hot D, et al: Dynamic compression of chondrocyte-agarose constructs reveals new candidate mechanosensitive genes. PLoS One 2012;7:e36964.

-21 Madej W, van Caam A, Blaney Davidson EN, van der Kraan PM, Buma P: Physiological and excessive mechanical compression of articular cartilage activates Smad2/3P signaling. Osteoarthritis Cartilage 2014;22:1018-1025.

-22 Madej W, van Caam A, Blaney Davidson EN, Hannink G, Buma P, van der Kraan PM: Ageing is associated with reduction of mechanically-induced activation of Smad2/3P signaling in articular cartilage. Osteoarthritis Cartilage 2016;24:146-157.
23 Brown SA, Pagani L, Cajochen C, Eckert A: Systemic and cellular reflections on ageing and the circadian oscillator: a mini-review. Gerontology 2011;57:427-434.

24 Dudek M, Gossan N, Yang N, Im HJ, Ruckshanthi JP, Yoshitane H, et al: The chondrocyte clock gene Bmall controls cartilage homeostasis and integrity. J Clin Invest 2016; 126:365-376.

25 Matta C, Mobasheri A: Regulation of chondrogenesis by protein kinase $\mathrm{C}$ : emerging new roles in calcium signalling. Cell Signal 2014; 26:979-1000.

26 Matta C, Mobasheri A, Gergely P, Zákány R: Ser/Thr-phosphoprotein phosphatases in chondrogenesis: neglected components of a two-player game. Cell Signal 2014;26:21752185.

27 Beier F, Loeser RF: Biology and pathology of Rho GTPase, PI-3 kinase-Akt, and MAP kinase signaling pathways in chondrocytes. J Cell Biochem 2010;110:573-580.

28 Kamata H, Hirata H: Redox regulation of cellular signalling. Cell Signal 1999;11:1-14.

29 Nelson KK, Melendez JA: Mitochondrial redox control of matrix metalloproteinases. Free Radic Biol Med 2004;37:768-784.

30 Dai SM, Shan ZZ, Nakamura H, MasukoHongo K, Kato T, Nishioka K, et al: Catabolic stress induces features of chondrocyte senescence through overexpression of caveolin 1 : possible involvement of caveolin 1-induced down-regulation of articular chondrocytes in the pathogenesis of osteoarthritis. Arthritis Rheum 2006;54:818-831.

-31 Yin W, Park JI, Loeser RF: Oxidative stress inhibits insulin-like growth factor-I induction of chondrocyte proteoglycan synthesis through differential regulation of phosphatidylinositol 3-kinase-Akt and MEK-ERK MAPK signaling pathways. J Biol Chem 2009; 284:31972-31981.
32 Oh CD, Chun JS: Signaling mechanisms leading to the regulation of differentiation and apoptosis of articular chondrocytes by insulin-like growth factor-1. J Biol Chem 2003; 278:36563-36571.

33 Starkman BG, Cravero JD, Delcarlo M, Loeser RF: IGF-I stimulation of proteoglycan synthesis by chondrocytes requires activation of the PI 3-kinase pathway but not ERK MAPK. Biochem J 2005;389:723-729.

34 Nishikawa T, Kukidome D, Sonoda K, Fujisawa $\mathrm{K}$, Matsuhisa T, Motoshima $\mathrm{H}$, et al: Impact of mitochondrial ROS production in the pathogenesis of insulin resistance. Diabetes Res Clin Pract 2007;77(suppl 1):S161-S164.

35 Campisi J: Senescent cells, tumor suppression, and organismal aging: good citizens, bad neighbors. Cell 2005;120:513-522.

36 Takahashi A, Ohtani N, Yamakoshi K, Iida S, Tahara H, Nakayama K, et al: Mitogenic signalling and the p16INK4a-Rb pathway cooperate to enforce irreversible cellular senescence. Nat Cell Biol 2006;8:1291-1297.

- 37 Zákány R, Szíjgyártó Z, Matta C, Juhász T, Csortos C, Szucs K, et al: Hydrogen peroxide inhibits formation of cartilage in chicken micromass cultures and decreases the activity of calcineurin: implication of ERK1/2 and Sox 9 pathways. Exp Cell Res 2005;305:190-199.

38 Steinberg GR, Kemp BE: AMPK in health and disease. Physiol Rev 2009;89:1025-1078.

39 Salminen A, Kaarniranta K, Kauppinen A: Age-related changes in AMPK activation: role for AMPK phosphatases and inhibitory phosphorylation by upstream signaling pathways. Ageing Res Rev 2016;28:15-26.

40 Liu-Bryan R: Inflammation and intracellular metabolism: new targets in OA. Osteoarthritis Cartilage 2015;23:1835-1842. 